\title{
Placing a text in context
}

\author{
DEBRA L. LONG \\ University of California, Davis, California \\ and University of Central Lancashire, Preston, England \\ AND \\ Alice Spooner \\ University of Central Lancashire, Preston, England
}

\begin{abstract}
Can readers accurately retrieve information about the context in which text comprehension occurs? If so, does their memory for context vary with their level of comprehension? Participants studied ambiguous passages in a high-knowledge or low-knowledge condition. They were then asked to remember the spatial location of individual sentences, the color of a border surrounding the passage, or the color of a shirt worn by the experimenter. Recall protocols were collected after participants answered the context question. Knowledge about the topic of the text facilitated both contextual retrieval and recall. Moreover, contextual retrieval and recall were correlated, primarily in the high-knowledge condition. The results suggest that personal experiences accompanying comprehension are encoded in memory along with text meaning and have implications for theories of source monitoring.
\end{abstract}

I know the answer to this question. I read it in my text yesterday at the library. I can see the page in my mind.

The experience described above is a familiar one. It involves our ability to retrieve the content of a text and the context in which reading occurred (e.g., time, location). Memory for context is often studied using sourcememory paradigms. Participants are asked to assign a recognized item to a context corresponding to the item's origin in memory (Johnson, Hashtroudi, \& Lindsay, 1993). For example, participants may be asked whether an item was presented in a particular spatial location, voice, or color. High rates of item memory are usually associated with high rates of context memory (Conway \& Dewhurst, 1995; but see Hornstein \& Mulligan, 2004).

The association between item memory and context memory is explained in an influential theory of episodic memory called the source monitoring framework (SMF) (Johnson et al., 1993). According to the SMF, an experience comprises many features: perceptual (e.g., color), spatial (e.g., location), semantic (e.g., category membership), and so on. These features can become bound during processing such that they make up a complex mental representation of an event. The extent of this binding determines the likelihood that one memory trace can be distinguished from another.

In most studies of source memory, the content of an item and the context in which it appears are part of the same perceptual event (Mitchell \& Johnson, 2009). Con- sider, for example, an experiment in which participants are asked to remember a list of words in colored font. The content of the word (its meaning) and the color in which it appears (context) are processed simultaneously. If participants are successful in binding the word with its color, retrieval of one feature will facilitate retrieval of the other.

In this article, we ask whether memory for the content of a text is associated with memory for the context in which reading occurred. This question is theoretically interesting, because it highlights an aspect of the SMF that is underspecified. The framework makes straightforward predictions about the association of content and context when context is a feature of the item's presentation, as it is in most studies of source monitoring. Its predictions are less clear, however, when the context is not a feature of the item. For example, a reader might recall ideas from a text and recall that he or she read the text sitting in a blue chair. The blue chair is an aspect of the context, but it is not a feature of the item per se.

Most theories of reading comprehension explain memory for the individual ideas in a text as a by-product of memory for the text as a whole (Kintsch, 1988, 1998). Texts have an embedded structure, such that individual ideas are integrated to form events and events are integrated to form still larger events. Text ideas are well remembered when readers have integrated them into coherent event structures (Zwaan, Magliano, \& Graesser, 1995). We ask whether the ability to integrate text ideas influences the reader's ability to retrieve contextual details about the reading episode when the text and the context

D. L. Long, dllong@ucdavis.edu 
can be processed simultaneously and when features of the context do not accompany presentation of the text.

The relation between text memory and context memory is also interesting from an empirical standpoint. To our knowledge, only one study has examined the issue. Cataldo and Oakhill (2000) had children read texts, then remember the spatial locations of individual words. Memory for location was unrelated to text comprehension. One possibility for the failure to observe a relation between comprehension and context memory is that single words are not the units of meaning encoded during comprehension. Readers retain the meaning of text ideas, but little about the surface form (e.g., individual words and word order). Thus, the association of content and context in comprehension may be apparent if we define content memory as memory for text ideas. In the following four experiments, we examined readers' ability to recall text ideas and their ability to retrieve contextual details of the reading episode.

\section{EXPERIMENT 1}

We manipulated text comprehension using a paradigm from a classic study by Bransford and Johnson (1972). Participants studied a passage containing numerous vague referring expressions. In the high-knowledge condition, background knowledge was provided to support com- prehension. In the low-knowledge condition, participants received either no relevant knowledge or too little information to disambiguate the references. We examined participants' recall of the text and their memory for the physical location of individual sentences as a function of knowledge. We predicted that the high-knowledge group would recall more ideas from the text than would the lowknowledge group, replicating Bransford and Johnson's findings. Our question was whether they would also be more accurate in identifying information about the spatial location of the sentences.

\section{Method}

Participants. Participants were 132 undergraduates in a methods course. Data from 2 participants were excluded because they did not answer the location question.

Materials and Procedure. The passages were from Bransford and Johnson (1972): "Washing Clothes" and "The Balloon." We used both passages to enhance the generalizability of our findings. In the high-knowledge condition, the "Washing Clothes" passage was presented with the title "Washing Clothes" and the "The Balloon" passage was presented with a cartoon that depicted the events described in the text. In the low-knowledge condition, the "Washing Clothes" passage was presented with no title and the "The Balloon" passage was presented with only a portion of the cartoon. A set of booklets was prepared; each contained one of the two passages, a set of anagrams (used in the distractor task), and a template of the passage corresponding to its physical layout (see the "Washing Clothes" passage and its template in Table 1). The template was presented

Table 1

The "Washing Clothes" Passage From Bransford and Johnson (1972), and the Spatial Layout Used in Experiment 1

\section{Washing Clothes}

The procedure is actually quite simple. First you arrange items into different groups. Of course one pile may be sufficient depending upon how much there is to do. If you have to go somewhere else due to lack of facilities that is the next step; otherwise, you are pretty well set. It is important not to overdo things. That is, it is better to do too few things at once than too many. In the short run, this may not seem important but complications can easily arise. A mistake can be made as well. At first, the whole procedure will seem complicated. Soon, however, it will become just another facet of life. It is difficult to foresee any end to the necessity for this task in the immediate future, but then, one never can tell. After the procedure is completed one arranges the materials into different groups again. Then they can be put into their appropriate places. Eventually they will be used once more and the whole cycle will have to be repeated. However, that is part of life.

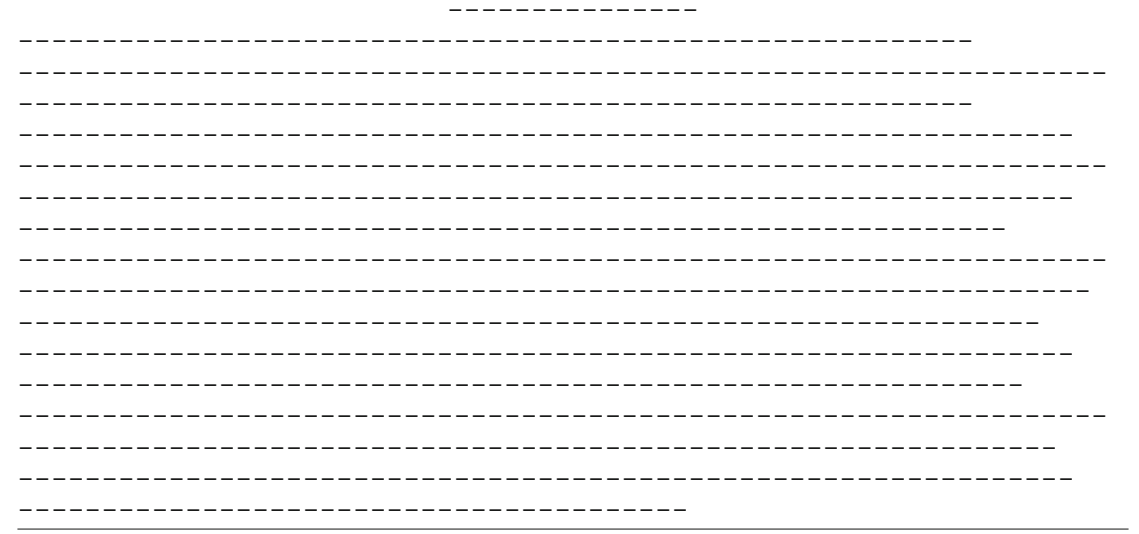


with a single test sentence. The test sentences varied across booklets in such a way that every sentence from a passage appeared as a test sentence except the first and last sentences. The passages and test sentences were counterbalanced across booklets.

The booklets were distributed randomly to students. They were told to read the passage carefully because they would be asked questions about it later. They were also told that the experimenter would instruct them when it was time to turn a page in the booklet and that they were not to turn back to previous pages. Students were given $5 \mathrm{~min}$ to study the passage. They then solved anagrams for $5 \mathrm{~min}$. Subsequently, they were asked about the spatial location of the test sentence. Participants placed an $\mathrm{X}$ at the location where the first letter of the first word had appeared in the original passage. They were then asked to recall the passage in as much detail as possible.

\section{Results and Discussion}

In all experiments, the context memory and recall measures were analyzed as a function of knowledge condition. We also conducted a regression analysis with recall as the dependent measure and knowledge condition, context retrieval, and the knowledge by context-retrieval interaction as predictor variables. Accuracy on the context questions for all experiments appears in Table 2. All effects were reliable at the $p<.05$ level unless indicated otherwise.

Memory for location. We computed three measures of location accuracy. Distance was the Euclidian distance (in millimeters) from the participants' $\mathrm{X}$ to the correct location. Additional measures were computed to examine the possibility that readers in the high-knowledge condition used knowledge rather than memory for the text to make their location judgments. Readers who knew the text was about a particular procedure (e.g., washing clothes) might identify a test sentence as a step that occurs early, midway, or late, then use this knowledge to locate the test sentence. This could be done in the absence of memory for the text. Although this strategy might help readers position a test sentence in the vertical direction, it should have no effect on their judgments in the horizontal direction. Thus, we computed a vertical measure - lines - as the number of lines between the indicated and the actual location and a horizontal measure - spaces - as the number of dashes between the indicated and correct location.

We found a reliable effect of knowledge on distance $\left[F(1,128)=7.05, M S_{\mathrm{e}}=1,076\right]$ and on spaces $\left[F(1,128)=5.49, M S_{\mathrm{e}}=146\right]$, but not on lines $(F<1)$. These findings suggest that comprehension contributed to high-knowledge readers' performance, because world knowledge alone provided no information about where sentences should be located in the horizontal direction.

Recall. We adopted the following procedure for scoring the recall protocols in all experiments. The original passages and protocols were divided into idea units, roughly corresponding to clauses, by two independent judges blind to knowledge condition. Recall score was the proportion of idea units from the passage that appeared in the protocol. Interrater reliability in Experiment 1 was 91\%. Readers in the high-knowledge condition recalled more ideas $(M=.59, S E=.03)$ than did readers in the lowknowledge condition $(M=.42, S E=.02)[F(1,128)=$ 19.01, $\left.M S_{\mathrm{e}}=.05\right]$.

Recall and context memory. We computed a regression equation with recall as the dependent variable and knowledge, distance, and the interaction between knowledge and distance as predictors. The three predictors accounted for a significant amount of variance in recall $\left[F(3,126)=22.21, M S_{\mathrm{e}}=.04\right]$. Recall was reliably predicted by knowledge $(t=5.51, B=.72)$, distance $(t=$ $-3.18, B=-.25)$, and the knowledge $\times$ distance interaction $(t=-3.98, B=-.50)$. Figure 1 depicts the interaction. Recall increased as distance decreased; however, the relation between recall and distance was stronger in the high-knowledge than in the low-knowledge condition.

The availability of relevant knowledge about the text improved both recall and memory for sentence location. In addition, recall and location memory were related more strongly in readers who had access to relevant knowledge during comprehension than in readers who lacked this knowledge. These findings are consistent with the claim

Table 2

Performance on Context Questions As a Function of Knowledge Condition for Experiments 1-4

\begin{tabular}{|c|c|c|c|c|c|c|}
\hline \multirow[b]{3}{*}{ Context Question } & \multicolumn{6}{|c|}{ Knowledge Condition } \\
\hline & \multicolumn{3}{|c|}{ High-Knowledge } & \multicolumn{3}{|c|}{ Low-Knowledge } \\
\hline & $M$ & $S E$ & $\%$ Correct & $M$ & $S E$ & $\%$ Correct \\
\hline \multicolumn{7}{|l|}{ Experiment 1} \\
\hline Distance (in $\mathrm{mm}$ ) & 31.52 & 2.99 & & 47.66 & 4.07 & \\
\hline Lines & 2.05 & 0.29 & & 2.22 & 0.20 & \\
\hline Spaces & 11.89 & 1.34 & & 17.13 & 1.44 & \\
\hline \multicolumn{7}{|l|}{ Experiment 2} \\
\hline Border color & & & 55 & & & 41 \\
\hline \multicolumn{7}{|l|}{ Experiment 3} \\
\hline T-shirt color & & & 39 & & & 27 \\
\hline \multicolumn{7}{|l|}{ Experiment 4} \\
\hline Distance (in $\mathrm{mm}$ ) & 30.55 & 3.20 & & 46.64 & 4.84 & \\
\hline Lines & 2.03 & 0.31 & & 2.00 & 0.28 & \\
\hline Spaces & 11.58 & 1.44 & & 17.97 & 2.02 & \\
\hline Border color & & & 58 & & & 28 \\
\hline Font color & & & 68 & & & 43 \\
\hline
\end{tabular}




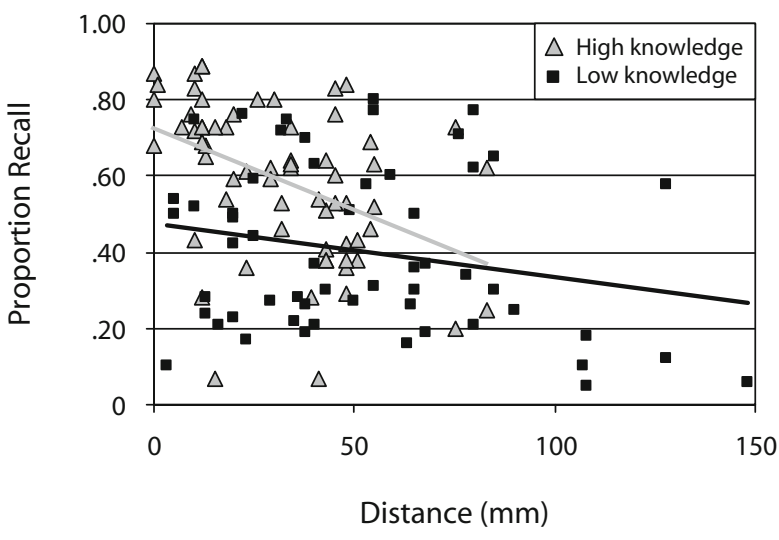

Figure 1. Proportion recall as a function of knowledge condition and distance judgments in Experiment 1.

that text comprehension enhances readers' retrieval of perceptual details of the reading experience.

\section{EXPERIMENT 2}

The meaning of a sentence and its location in a text is somewhat related in procedural texts like those used in this study; the order of sentences is associated with steps in the procedure. In Experiment 2, we investigated text memory and context memory when meaning and context were unrelated. Participants were asked to remember the color of a border that surrounded the text. The relation between color and text content was arbitrary.

\section{Method}

Participants, Materials, and Procedure. Participants were 251 undergraduates in an introductory psychology course. The materials and procedure were identical to those in Experiment 1, with the following exceptions: (1) Each passage was printed on a page with a thin border (3 pt.; red, blue, green, or yellow) along the outer edges, and (2) the context question asked readers to recall the color of the border. They were given four alternatives (red, blue, green, yellow).

\section{Results and Discussion}

Interrater reliability in scoring the recall protocols was 93\%. Readers in the high-knowledge group recalled more ideas $(M=.51, S E=.02)$ than did readers in the lowknowledge group $(M=.41, S E=.02)[F(1,249)=13.28$, $\left.M S_{\mathrm{e}}=.05\right]$. The high-knowledge group was also more accurate at remembering the color of the border than was the low-knowledge group; see Table 2 for the percent cor$\operatorname{rect}\left[\chi^{2}(1)=5.13\right]$.

We examined the extent to which recall was related to knowledge and accuracy on the context question, using the same procedure as we did in Experiment 1. The predictor variables accounted for a significant amount of variance in recall $\left[F(3,247)=15.22, M S_{\mathrm{e}}=.04\right]$. The only reliable predictor was the knowledge $X$ accuracy interaction $(t=2.31, B=.25)$. The association between recall and accuracy was stronger in the high-knowledge than in the low-knowledge condition. The interaction is depicted in Figure 2.
These data extend the results of Experiment 1 by showing that comprehension, facilitated by the activation of background knowledge, influenced both text memory and context memory. This occurred even though the context information was incidental and unrelated to the passage meaning.

\section{EXPERIMENT 3}

In Experiments 1 and 2, the contextual information was temporally and spatially associated with the passage; it appeared at the same time and on the same page. The results of these experiments can be explained in light of existing theories of source memory. An item (e.g., a word or sentence) appears in a particular context (e.g., spatial location, or with a surrounding border). The item and the context are processed simultaneously and may be linked in memory; thus, any improvement in item memory will be associated with an improvement in context memory.

Our claim, however, is that item memory in text comprehension (i.e., memory for individual sentences) is secondary to memory for the text as a whole. Sentences are embedded in texts and texts are embedded in contexts. Thus, the text itself may be an episodic unit in memory and may be associated with a context; this might occur even if the contextual information appeared separately from the text ideas in space and time. We investigated this claim in Experiment 3 by asking students to remember a contextual detail of the experiment that occurred before the text was presented.

\section{Method}

Participants, Materials, and Procedure. Participants were 238 undergraduates in a methods course. The materials and procedure were identical to those in Experiment 1, except for the following. An experimenter distributed test booklets in the students' classroom and explained the task, then left the room and the class instructor asked participants to begin the task. After studying the passage and performing the distractor task, participants were asked to remember the color of the shirt worn by the experimenter when she distributed the test booklets. Participants were given four alternatives (red, blue, green, yellow).

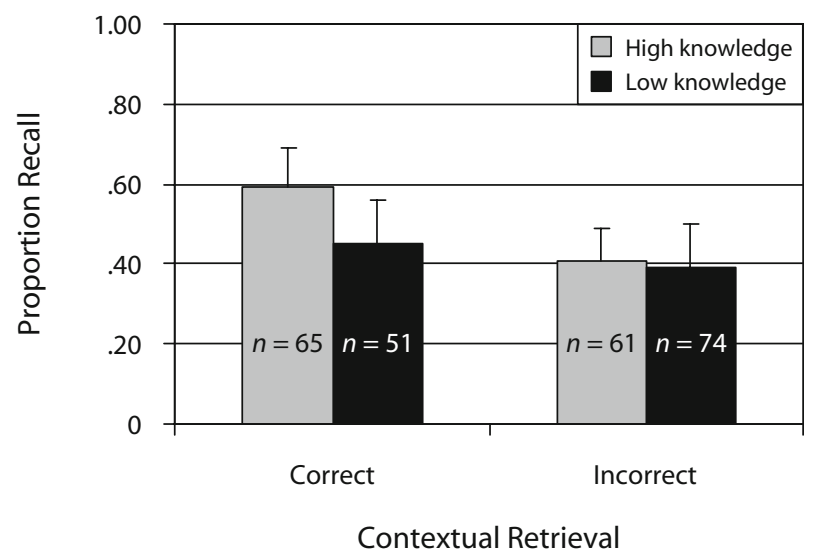

Figure 2. Proportion recall as a function of knowledge condition and accuracy on the context question in Experiment 2. 


\section{Results and Discussion}

Interrater reliability in scoring the recall protocols was $88 \%$. Readers in the high-knowledge group recalled more ideas $(M=.52, S E=.02)$ than did readers in the lowknowledge group $(M=.42, S E=.02)[F(1,236)=13.88$, $\left.M S_{\mathrm{e}}=.04\right]$. In addition, the high-knowledge group was more accurate at remembering the color of the experimenter's shirt; see Table 2 [ $\left.\chi^{2}(1)=4.07\right]$.

We conducted the regression analysis as in Experiment 2 . The predictors accounted for a reliable amount of variance in recall $\left[F(3,234)=7.41, M S_{\mathrm{e}}=.04\right]$. Knowledge and the knowledge $\times$ accuracy interaction were both marginally reliable $(t=1.85, p=.07, B=.14$; and $t=$ $1.8, p=.07, B=.20$, respectively). Recall and context memory were more strongly related in the high-knowledge than in the low-knowledge condition. The interaction is depicted in Figure 3.

These data extend our previous results by showing a general enhancement of memory associated with background knowledge. Readers who had relevant knowledge about the text had high recall and above-chance memory for the color of the experimenter's shirt, even though the experimenter was not physically present when the passage was comprehended. Moreover, only those readers in the high-knowledge condition showed a relation between recall and memory for context.

\section{EXPERIMENT 4}

In the previous experiments, participants were given a fixed time for studying the passage to control for the interval between comprehension and recall. One problem with this design is that the high-knowledge group is likely to have read the passage more quickly than the lowknowledge group did. This raises the possibility that they used the remaining study interval to employ strategies in which they linked the text to the context. We examined this possibility by having participants in Experiment 4 read the passage at their own rate, then immediately respond to the context questions.

We assessed memory for contextual details that were the same as or analogous to those used in Experiments 1-3.

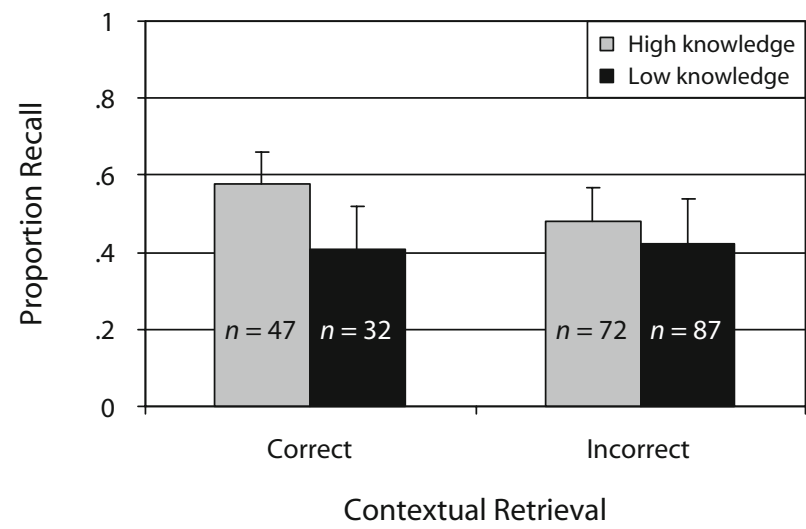

Figure 3. Proportion recall as a function of knowledge condition and accuracy on the context question in Experiment 3.
Participants were asked to report the spatial location of a test sentence and to report the color of a border that surrounded the text. We also asked participants to recall the color font used in a welcome message that appeared at the beginning of the experiment before readers received the passage. This measure is similar to the one used in Experiment 3 ("recall the color of the experimenter's T-shirt"), in that both events were unrelated to the passage meaning and occurred before the passage was read.

\section{Method}

Participants, Materials, and Procedure. Participants were 80 undergraduates who received course credit for their participation. The passages, knowledge conditions, and distractor task were the same as those used previously. The passages were presented with a thin colored border, as in Experiment 2.

The experiment began with a welcome message displayed on a computer screen in one of four colored fonts (red, blue, green, or yellow). Participants pressed the Enter key at the experimenter's request; the welcome screen disappeared, and instructions appeared. After reading the passage, participants received the distractor task, then a set of three context questions, presented in random order. One question asked for the location of a test sentence; a second asked participants to identify the color of the border surrounding the passage. The third question asked them to identify the font color used in the welcome message that preceded the experiment. Participants were given four options (red, blue, green, yellow). The context questions were followed by the recall task.

\section{Results and Discussion}

Interrater reliability in scoring the recall protocols was $87 \%$. The high-knowledge group recalled more ideas $(M=.60, S E=.02)$ than the low-knowledge group did $(M=.44, S E=.02)\left[F(1,79)=11.55, M S_{\mathrm{e}}=.04\right]$. The high-knowledge group was also more accurate at answering the context questions (see Table 2). We found reliable effects of knowledge on distance $\left[F(1,79)=7.98, M S_{\mathrm{e}}=\right.$ $614.62]$, and spaces $\left[F(1,79)=6.85, M S_{\mathrm{e}}=113.20\right]$, but not on lines $(F<1)$. We also found a knowledge effect on border color $\left[\chi^{2}(1)=14.73\right]$ and font color $\left[\chi^{2}(1)=\right.$ 8.21].

Reading times. As expected, the high-knowledge group had faster reading times than did the lowknowledge group $(M=5,637 \mathrm{msec}, S E=132.17$; and $M=6,395 \mathrm{msec}, S E=172.92$, respectively) $[F(1,79)=$ $\left.12.11, M S_{\mathrm{e}}=947,364\right]$. The correlation between reading time and recall was not significant $(r=-.15)$. We used reading time, knowledge condition, and the interaction of reading time and knowledge to predict performance on the context questions. Distance was analyzed by means of a regression analysis; border color and font color were analyzed by means of logistic regression. Knowledge was a reliable predictor of distance, border color, and font color ( $b=-2.83,3.57$, and 2.81, respectively). We found no other reliable effects.

We conducted this experiment to determine whether study time could explain the relation between text memory and context memory. We suggested that high-knowledge readers may have spent less time processing the passages than did low-knowledge readers, and that they used the remaining study time to think about aspects of the context. Our findings are inconsistent with this hypothesis. 
Although high-knowledge readers comprehended the passages faster than low-knowledge readers did, they had no opportunity for additional study, because the context questions were presented immediately after comprehension.

\section{GENERAL DISCUSSION}

Can readers accurately retrieve information about the context in which comprehension occurs? If so, does their memory for context vary with their level of comprehension? We found that readers who were given knowledge to aid comprehension recalled more text ideas than did readers who received insufficient knowledge, replicating the classic Bransford and Johnson (1972) findings. More important for the goal of this study is our finding that knowledge also enhanced memory for the context in which the text appeared.

The results that we obtained in Experiments 1 and 2 are readily understood in light of the SMF (Johnson et al., 1993). Sentences in the text were accompanied by the contextual features that we tested (location and border color, respectively). Participants in the knowledge condition represented text ideas more strongly than did participants in the no-knowledge condition; thus, these participants had richer, more embellished representations. According to the SMF, features of an item, including its context, are bound into an integrated representation in such a way that retrieval of the memory trace involves retrieval of both content and context.

The SMF provides a less straightforward account of our findings in Experiments 3 and 4. We tested participants' memories for a contextual detail that occurred before the text was presented (e.g., the color of the experimenter's shirt and the font color of a welcome screen). How were content and context bound when their features did not co-occur?

We suggest that this question may be answered by considering how texts, and events more generally, are structured in memory (Zacks, Speer, Swallow, Braver, \& Reynolds, 2007). Events can be defined as segments of time that have a beginning and an end. They are represented at multiple levels (time frames) and are organized hierarchically. Events are clustered or "chunked" into increasingly larger events with longer time frames. Recent research suggests that readers are very sensitive to the structure of events in a text (see Zacks et al., 2007, for a review). For example, reading times are longer for sentences at event boundaries than for nonboundary sentences, suggesting that readers update their text representations to reflect the transition from one event to another. In addition, text ideas that appear at event boundaries are better remembered than those that appear before or after a boundary.

The embedded structure of events in memory may help to explain our results in Experiments 3 and 4. In these experiments, individual text ideas were embedded in events that made up the text, and the text as a whole was embedded in an experiment. Participants in the high-knowledge condition, relative to those in the low-knowledge one, may have constructed a more coherent event structure in which individual text ideas were integrated with each other and with the overall topic of the text. Memory for the text as a whole might then have facilitated memory for the event in which comprehension was embedded (e.g., the experiment). If this explanation is correct, it has implications for our understanding of source monitoring. Memory for the context of an event will depend on two factors: the extent to which features of an item are bound together during processing, as described explicitly in SMF; and the extent to which an item is embedded in an event hierarchy. When an item in an event is well remembered, features of the context in which the event was embedded will also be well remembered.

In summary, readers who have understood a text are likely to remember aspects of the context in which comprehension occurred. Our results are the first empirical demonstration that text comprehension facilitates both text memory and the retrieval of contextual details about the reading experience. The ability to remember the content of a text is a reliable indicator of a reader's ability to place a text in context.

\section{AUTHOR NOTE}

This study was supported by grants from the National Science Foundation (NSF0231152) and the National Institutes of Health (R01HD489141A2). We thank Andrew Yonelinas for his helpful discussions on this topic. Correspondence concerning this article should be sent to D. L. Long, Department of Psychology, University of California, 1 Shields Avenue, Davis, CA 95616 (e-mail: dllong@ucdavis.edu).

\section{REFERENCES}

Bransford, J. D., \& Johnson, M. K. (1972). Contextual prerequisites for understanding: Some investigations of comprehension and recall. Journal of Verbal Learning \& Verbal Behavior, 11, 717-726.

Cataldo, M. G., \& OAKhill, J. (2000). Why are poor comprehenders inefficient searchers? An investigation into the effects of text representation and spatial memory on the ability to locate information in a text. Journal of Educational Psychology, 92, 791-799.

Conway, M. A., \& Dewhurst, S. A. (1995). Remembering, familiarity, and source monitoring. Quarterly Journal of Experimental Psychology, 48A, 125-140.

Hornstein, S. L., \& Mulligan, N. W. (2004). Memory for actions: Enactment and source memory. Psychonomic Bulletin \& Review, 11, 367-372.

Johnson, M. K., Hashtroudi, S., \& Lindsay, D. S. (1993). Source monitoring. Psychological Bulletin, 114, 3-28.

KInTSCH, W. (1988). The role of knowledge in discourse comprehension: A construction-integration model. Psychological Review, 95, 163-182.

KInTsch, W. (1998). Comprehension: A paradigm for cognition. New York: Cambridge University Press.

Mitchell, K. J., \& Johnson, M. K. (2009). Source monitoring 15 years later: What have we learned from fMRI about the neural mechanisms of source memory? Psychological Bulletin, 135, 638-677.

Zacks, J. M., Speer, N. K., Swallow, K. M., Braver, T. S., \& ReynOLDS, J. R. (2007). Event perception: A mind/brain perspective. Psychological Bulletin, 133, 273-293.

Zwann, R. A., Magliano, J. P., \& Graesser, A. C. (1995). Dimensions of situation model construction in narrative comprehension. Journal of Experimental Psychology: Learning, Memory, \& Cognition, 21, 386-397.

(Manuscript received October 13, 2008; revision accepted for publication November 2, 2009.) 